\title{
Editorial
}

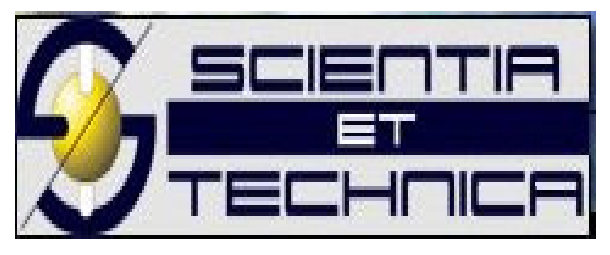

\section{Una mirada a la convocatorio de Publindex - Colciencias}

Continuando con el análisis sobre la convocatoria hecha por Colciencias para indexar las revistas científicas podemos recurrir a lo que ha sucedido en el tiempo, para ir mirando la trayectoria de los procesos de indexación de revistas, por los menos en los últimos 6 años. En este sentido, si referenciamos la convocatoria realizada en el año 2013, en donde participaron un total de 565 y de acuerdo a los criterios de calidad científica y editorial $\mathrm{y}$ según los perfiles de estabilidad y visibilidad del Servicio Permanente de Indexación de Revistas Colombianas especializadas en Ciencia y Tecnología fueron indexadas 515 , posteriormente en el año 2014, prácticamente con los mismos criterios de selección, de las 558 revistas que participaron en la convocatoria, fueron indexadas 542. [1]

Sin embargo, en el año 2015 como ya se ha publicado y los hemos manifestado en varias ocasiones, Colciencias inició una estrategia para rediseñar el Publindex, con el fin de construir un sistema de valoración de las revistas nacionales a partir de criterios existentes y comúnmente 
aceptados para evaluar su gestión editorial, calidad de contenido, nivel de citación, regularidad y accesibilidad.

Recordamos entonces, que el objetivo principal de esta última convocatoria, busca evaluar la calidad de las revistas científicas nacionales, mediante criterios relacionados con la gestión editorial, visibilidad e impacto de las publicaciones, además se busca generar indicadores confiables sobre las capacidades de SNCTeI en cuanto a las revistas cientificas nacionales, a partir de información validada y actualizada: simultáneamente se pretende implementar nuevos mecanismos para mejorar su clasificación, visibilidad e impacto.

Es de notar, que de acuerdo a estudios hechos por Colciencias hay un evidente desbalance en Colombia que repercute en la falta de calidad y visibilidad de las revistas nacionales, básicamente y concluyendo al respecto, se puede decir que en la gran mayoría de países extranjeros y con una cuota alta en los proceso de investigación, existen un gran número de revistas científicas de impacto con un buen número de investigadores que escriben en ellas, por ejemplo en España hay alrededor de 1000 revistas científicas, pero hay 120.000 investigadores a diferencia de nuestro país donde encontramos 542 revistas reconocidas por el IBN Publindex, clasificadas en A1, A2, B y C para sólo 10.000 investigadores.[2]

De 10 anterior, es de notar también, que solo 75 de estas revistas se encuentran en los principales índices bibliográficos citacionales, Journal Citation Report (JCR) y SCImago Journal Rank (SJR). Lo anterior evidencia el pobre aporte de Colombia al total de la producción científica internacional en publicaciones de alto impacto.

En realidad, no se puede dudar de que la estrategia que Colciencias busca con esta nueva convocatoria es aumentar la participación de los investigadores nacionales en revistas científicas indexadas en indices citacionales de alto impacto: Sin embargo para ello es necesario que se cumpla con nuevos mecanismos para apoyar a los investigadores en el proceso de sometimiento de sus artículos en las revistas con el objetivo de dinamizar e incrementar la participación de investigadores en proyectos de investigación que involucren al mismo tiempo pares internacionales.

Por lo anterior y como consecuencia de estas nuevas estrategias es posible la desaparición de un gran número de revistas que se encuentran actualmente en el IBN, dicha situación, se convierte en un motivo más de preocupación para los editores de nuestras revistas nacionales, ya que la disminución de revistas en nuestro medio va generar un impacto negativo en el trabajo que realizan los grupos de investigación y obviamente en nuestros investigadores. 
Hugo Armando Gallego B.

Editor en Jefe, Revista Scientia et Technica, Docente de Planta, Universidad Tecnológica de Pereira, Pereira, Risaralda, Colombia.

E-mail: ugo@utp.edu.co

\section{Referencias}

[1]. http://www.mineducacion.gov.co/

[2]. http://www.colciencias.gov.co/ 